\title{
Exophthalmos as the first manifestation of mantle cell lymphoma of the patient with Hashimoto thyroiditis.
}

Authors: Krzysztof Marczewski ${ }^{1,2}$, Dorota Boniek-Poprawa1 ${ }^{1}$, Piotr Tomaszewski ${ }^{1}$, Agnieszka Kawałko ${ }^{1}$, Marek Maciejewski ${ }^{1}$

1. Department Nephrology, Endocrinology John Paul The II Regional Hospital in Zamość

2. The Faculty of Medicine Lublin University of Economy and Innovation

\section{OBJECTIVES}

The diagnosis of thyroid orbithopathy is based on clinical symptoms and typical changes in laboratory tests confirming thyroid disease. In approximately $5 \%$ it is Hashimoto's thyroiditis. Typical treatment is primarily steroids administered intravenously. But one disease does not exempts the other, and the drugs can not read. Therefore, we would like to present the history of our patient.

\section{CASE REPORT}

55 year-old man was admitted to the Endocrinology Ward due to a suspected thyroid orbitopathy, with the intention of steroid pulse therapy. The patient for a few weeks remained swollen eyelids, watery eyes, protrusion of eyeballs.

On physical examination, otherwise no significant deviations from the norm.

They showed normal levels of TSH, FT3 and FT4, and TR AB, and AB TPO exceed the norm of 25.3 (N 0-9 IU / $\mathrm{ml})$, with of other tests drew our attention to a small lymphocytosis $(5, \mathrm{G} / \mathrm{l})$.

$\mathrm{MRI}$ of the orbits revealed on both sides surrounded eyeballs confluent, well demarcated areas undergoing moderate hypo-intensive strengthening mainly in the area of mine-medial orbital segments. The muscles of the correct size. The eyeballs symmetrical correct size and shape, moved slightly forward. That are likely infiltration of the type of lymphoma, inflammatory changes less likely. The ultrasound said enlarged lymph nodes in the neck bilaterally largest $20 \times 10 \mathrm{~mm}$ on the right side, left side $19 \times 8 \mathrm{~mm}$, near the axillary node on the right size $25 \times 14 \mathrm{~mm}$, on the left the size of $26 \times 12 \mathrm{~mm}$.

The patient was taken right axillary node for pathology, which was a mantle cell lymphoma classical type. The patient was referred for further treatment to the Clinic of Hematology.

\section{Conclusion}

If in our patient's treatment was based on clinical features of orbithopathy, then most likely it would lead to a initial clinical improvement because lymphoma respond to treatment with corticosteroids. However, this delay proper diagnosis and treatment, and probably was detrimental to the patient.

Despite some similarities in histology this is the first case known to us coexistence of mantle cell lymphoma and Hashimoto thyroiditis 